\title{
Chemotherapy for carcinoma of the bronchus-a brief review
}

\author{
NORMAN M. BLEEHEN \\ M.A., B.SC., F.R.C.P., F.F.R. \\ Professor of Radiotherapy, University of London
}

\begin{abstract}
Summary
A brief theoretical approach to the chemotherapy of carcinoma of the bronchus is considered. Some of the currently available data and prospects for the future are reviewed.
\end{abstract}

\section{Introduction}

Chemotherapy is less favoured as a general method of treatment of cancer than surgery or radiotherapy, and unfortunately this review will be more concerned with hopes for the future than present achievements. However, reference to some other diseases might indicate that this optimism is not without some justification. There have been several recent symposia and reviews on the management of carcinoma of the lung by chemotherapeutic agents (e.g. Carter, 1972; Holsti, 1962; Higgins, 1972; Selawry, 1970), and this review will not attempt to make a comprehensive survey of the subject. Some brief indications of the general scope of chemotherapy will be mentioned, and then some of the aspects that apply to cancer of the lung will be considered.

\section{Chemotherapy target}

Surgery and radiotherapy have a localized approach to a disease. Systemic therapy, as is effected by a chemotherapeutic regime, is potentially a useful treatment concept when one considers bronchial carcinoma. Chemotherapeutic agents will act systemically throughout most tissues. The main limiting factor will be impermeable parts of the tumour, and privileged sites that might be protected by physiological restraint, such as the blood brain barrier. This widespread action might then have a useful effect on metastatic disease, in addition to that on the primary lesion.

When one considers a strategy for their use, a brief initial theoretical approach may be of some value. As a simple generalization, a constant proportion of cells will be killed for equal doses of the drug, assuming equal accessibility and similar sensitivity of all the cells in the tumour to that drug. So that if one is treating with a dose of an agent which will kill $10 \%$ of the cells present, one will then kill $10 \%$ whether one is starting off with $10^{11}$ or $10^{9}$ or only
10 cells. This is, of course, a gross oversimplification, but one can see therefore that a useful gain might be achieved if one were to remove the majority of the tumour first by other means such as surgery or radiotherapy. A major factor which limits the usefulness of cytotoxic agents is their effect on rapidly proliferating normal tissues such as the bone marrow and the intestine. If it were possible to reduce the primary tumour mass to below a critical number of cells, one then would have a better chance of eliminating the remaining tumour cells by chemotherapy. Fig. 1 diagrammatically illustrates the theoretical difference that may exist between a patient who has a cancer which usually remains localized, with metastatic disease only occurring

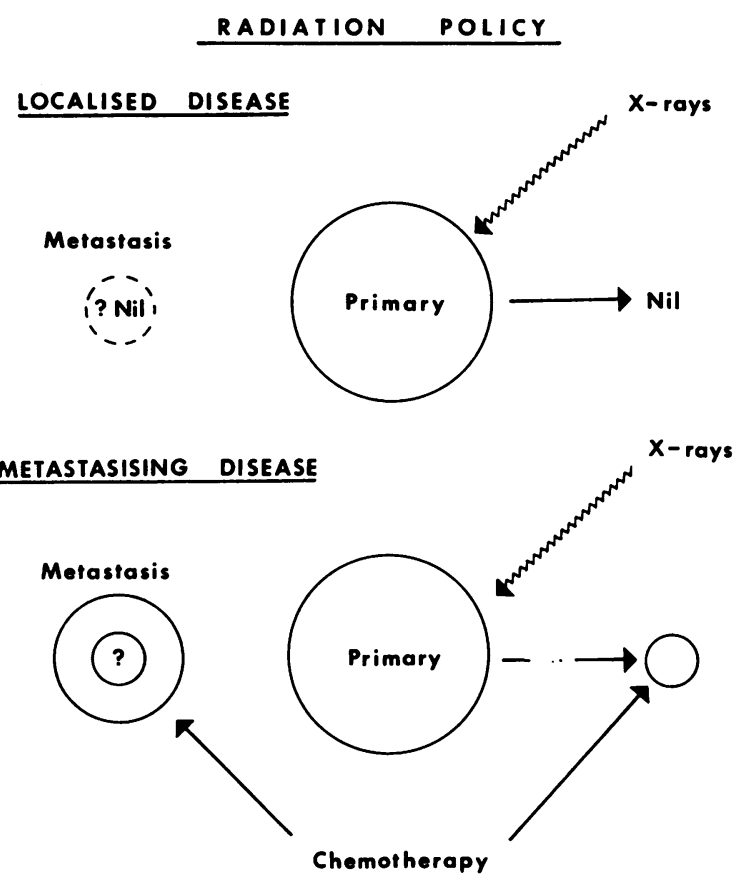

FIG. 1. The upper part of the figure shows the treatment policy for localized cancer. The lower part shows the altered role of radiotherapy in reducing tumour bulk to an amount that may then be controlled by subsequent chemotherapy. 
late in its clinical course; and a patient with carcinoma of the lung associated with poor prognostic factors, where there may be as much tumour in peripheral metastases as exists at the primary site. The removal of a large part of the primary lung tumour either by palliative radiotherapy or palliative surgery would have as much therapeutic value as a more radical approach to the local disease which was associated with greater morbidity. One can perhaps create a concept of critical cell kill at the primary site beyond which it is not worth attempting radical local therapy. Chemotherapy schedules may then be used to treat both the residual primary disease and the metastatic disease.

\section{Drug combinations}

Many of the drugs which have been discovered are thought to act at different biochemical sites within the cell. Some of these, like the alkylating agents, are thought to be almost proliferation independent and will act on the cells with the same general effect throughout all the stages of the cell cycle. Others may be either partially or wholly cell proliferation dependent. The toxic effects also may vary and it is by judicious use of these various groups of drugs that the combinations of treatment are built up.

This has been particularly useful in the leukemias and lymphomas, where the prognosis for acute lymphatic leukemia has changed considerably over the last few years, mainly due to this increasing awareness of the value of combination of drugs and the critical timing of the order of these drugs. Likewise the introduction of a multiple therapy regime for the lymphomas has resulted in a considerable improvement in the treatment of those diseases (discussed by Frei, 1972). Unfortunately, when one comes to other solid tumours the position is not quite so hopeful. This may be that the correct agents have not yet been discovered, and in part that the use of the existing ones has not been optimized.

\section{Single agents for carcinoma of the bronchus}

The chemotherapy of carcinoma of the bronchus is by no means as promising as for many other tumours that have been studied. Responses to single agents are observed but they are not very frequent and are unsustained.

Table 1 lists these in three groups which represent varying degrees of objective response as reported by various authors and summarized by Carter (1972). The group of agents with responses of $20 \%$ or more is perhaps the most interesting one from the point of view of the treatment of lung cancer and contains the ones that are most likely to build up into useful combinations, although this does not preclude useful effects in combination from other agents which have
TABLE 1. Response to chemotherapy for various drugs

\begin{tabular}{|c|c|c|}
\hline \multicolumn{3}{|c|}{ Percent responses to chemotherapy } \\
\hline Less than $10 \%$ & $10-19 \%$ & $20 \%$ or more \\
\hline Chlorambucil & $\begin{array}{l}\text { Phenyl alanine } \\
\text { mustard }\end{array}$ & Nitro Mustard \\
\hline $\begin{array}{l}\text { 6-Mercaptopurine } \\
\text { 5-Flurouracil } \\
\text { Vincristine } \\
\text { Cytoxine arabinoside } \\
\text { Actinomycin D } \\
\text { Bleomycin } \\
\text { Mithramycin }\end{array}$ & $\begin{array}{l}\text { Vinblastine } \\
\text { Hydroxyurea } \\
\text { Procarbazine } \\
\text { BCNU* }\end{array}$ & $\begin{array}{l}\text { Busulphan } \\
\text { Cyclophosphamide } \\
\text { Methotrexate } \\
\text { Adriamycin } \\
\text { Mitomycin C } \\
\text { CCNU* }^{*}\end{array}$ \\
\hline
\end{tabular}

* Nitrosurea drugs.

Data summarized from Carter (1972).

only been tested singly. It is worth remembering that these responses should be compared with the majority of patients with localized disease who will show a local response to radiation therapy. It is also worthy of note that a response observed using a particular drug regime may make little impression on the overall median survival of groups of patients with carcinoma of the lung so treated. A high percentage of responders, using a particular regime, is not necessarily associated with any increased median duration of that response (see Table 16 in Selawry, 1970).

A comparative review of some of the previous studies of chemotherapy for carcinoma of the lung is difficult because the range of chemosensitivity is great. It is generally agreed that small round cell (oat cell) carcinoma is more sensitive than the other types (Carbone et al., 1970). This cell type represents only $10-20 \%$ of the total number of cases seen but is an important target group for chemotherapy. For example, Hansen \& Muggia (1972b) have shown that almost half of these patients have marrow involvement at the time of presentation. That the primary site may be responsive to radiotherapy, is shown by the results of the Medical Research Council study (1969). Rissanen, Tikka \& Holsti (1968) showed that $35 \%$ of such patients may die without autopsy evidence of disease in the primary area. Any chemotherapeutic measures controlling peripheral meta. static disease might therefore be expected to improve the survival statistics. Green et al. (1969) reported an increase in mean survival from 1.8 months for placebo treatment to 5.6 months for patients treated with intermittent courses of Cyclophosphamide. This was highly significant statistically. However, the response of other cell types, as measured by survival, was poor. When the whole group of histological types was compared together for placebo and Cyclophosphamide, the results of 2.0 and 2.7 months respectively, obscured the specific value of the drug regime in oat cell tumours. A similar conclusion might be reached for the effect of nitrogen mustard 
on moderately and well differentiated squamous carcinoma, as seen in their study.

\section{Surgery plus adjuvant chemotherapy}

The role of adjuvant chemotherapy in the surgical management of carcinoma of the lung has been summarized recently by Higgins (1972), with particular reference to those studies that were carried out at the Veterans Administration Hospitals in the USA. In an early study, the role of Nitrogen Mustard given at the time of surgery and on the first and second post-operative days was investigated. There was no significant difference in the survival of the drug treated and control groups. A similar trial substituted Cyclophosphamide for Nitrogen Mustard and also showed no benefit. It seemed that the patients with oat cell carcinoma did respond somewhat better to the chemotherapy; with a $16 \%$ fouryear survival in the drug treated, as opposed to $4 \%$ four-year survival in the control groups. However, these results were not statistically significant. Brunner, Marthaler \& Muller (1971) reported a somewhat similar study investigating the adjuvant effect of Cyclophosphamide on radically operated bronchial carcinoma. They found that the mean survival time from operation to recurrence and from recurrence to death was reduced in the patients receiving the adjuvant chemotherapy. These results were also not statistically significant. However, the cumulative incidence of recurrence was significantly different at all the times reported, being adversely affected by the chemotherapy. They used a protracted regime with the total chemotherapy lasting for up to 2 years. They speculated that their adverse results may have been due to a continuous degree of immunosuppression with consequent deleterious side effects. The Medical Research Council Lung Cancer Working Party (1971) in a rather similar study compared the effects of Busulphan or Cyclophosphamide with a placebo on the post-operative survival. No difference was observed during the period of review. Higgin (1972) has given some early details of a study using a multiple drug regime. Post-operative patients who have no microscopic evidence of residual disease but who have certain criteria thought to be associated with a poor prognosis have been randomized to regimes receiving Cyclophosphamide in intermittent courses either alone or alternating with Methotrexate. The results are not yet available.

\section{Adjuvant chemotherapy plus radiation treatment}

The situation with regard to a combination of chemotherapy and radiotherapy is equally confusing and no clear cut position can be stated at this time.

Several studies combining the effect of an alkylating agent with radiation have not yielded any very significant results (see Holsti, 1972). The halogenated pyrimidine, 5-Flurouracil (5FU) is another agent which has been investigated frequently in this context. The most significant result was obtained by Gollin, Ansfield \& Vermund (1964). In a randomized controlled trial they found that the mean survival of thirteen patients treated by radiation alone was 6.2 months and radiation plus 5FU was 24 months. However, this particular trial was discontinued because of two haematological deaths, and when it was repeated at a lower dose of 5FU it failed to show any very significant result.

One other study should be mentioned which is of interest. Coy (1970) reported a randomized trial of irradiation and Vinblastine in lung cancer. Nearly 200 cases were included in the study but no significant difference in survival was found between the two groups. Radiation was given in the chemotherapy group $\frac{1}{2}$ hr after a dose of Vinblastine. A similar attempt at this sort of drug scheduling is reported by Frei (1972) using Vincristine in conjunction with Bleomycin, instead of radiation. Bleomycin on its own has not been usually found to have a very useful effect and Vincristine is only moderately effective. In this particular study, Bleomycin given at an interval after Vincristine yielded a relatively high number of partial responses, albeit in a small number of patients.

Bersagel and his colleagues (Bersagel et al., 1972) recently reported on a randomized study from Canada. Non-resectable carcinoma confined to the central chest was randomized into three groups, each receiving a course of radiotherapy with either no chemotherapy, four, or eight courses of Cyclophosphamide given every 3 weeks. The median survival of the radiotherapy group alone was 216 days and of the other two groups 306 days. They therefore demonstrated that the addition of Cyclophosphamide in this particular dose schedule of $1 \mathrm{~g} / \mathrm{m}^{2}$ intravenously every 21 days was of some minimal value.

\section{Multiple drug combinations}

More hope is aroused by the use of multiple drug regimes. Hansen and his colleagues (Hansen et al., 1972a) have reported on a randomized study of intensive combined chemotherapy in association with radiotherapy for patients with unresectable carcinoma of the bronchus. The treatment schedule was complex, involving a sequence of pairs of chemotherapeutic agents. These pairs of drugs were Cyclophosphamide plus Methotrexate, Actinomycin D plus Vincristine (together with radiation) and CCNU plus hydroxyurea. The patients were staged in great detail using currently available techniques and tumour histology was recorded. The reșults were correlated according to the staging. Limited disease patients, that is where disease was located within one hemithorax, had a better prognosis than 
those with more extensive disease. The median survival of patients with limited disease was 13 months as opposed to 4 months for those with extensive disease. Responders to chemotherapy lived significantly longer than non-responders with a median survival of 365 days as opposed to 130 days. This was not a randomized study and it is rather difficult to know how much influence this chemotherapy had on survival other than the somewhat artificial grouping into responders and non-responders. The response rate was highest and the survival longest in patients whose histology was either oat cell carcinoma or adenocarcinoma. The main interest of this particular study was that it showed that intensive chemotherapy could be tolerated by the patient and gives hope for useful results from other multiple drug regimes.

It is hoped that suitable combinations of drugs will improve the median survival and quality of survival. The most promising drugs under investigation at present include Methotrexate, Cyclophosphamide, Vincristine and nitrosourea derivatives (BCNU, CCNU and MeCCNU) in various combinations. The nitrosourea derivatives, which are lipid soluble, may be included in such a regime because of their effect on brain metastases by reason of easy passage across the blood brain barrier. Numerous other drugs, currently under early study (Carter, 1972) will become available for trial in various combinations. However, at the moment one can only have guarded optimism for the future.

\section{References}

Bersagel, D.E., Jenkins, R.D.T., Pringle, J.F., White, D.M., Fetterleg, J.C.M., Klassen, D.J. \& MCDermot, R.S.R. (1962) Lung Cancer. Clinical trial of radiotherapy alone vs radiotherapy plus cyclophosphamide. Cancer, 30, 621 .
Brunner, K.W., Marthaler, Th. \& Muller, W. (1971) Unfavourable effects of long-term adjuvant chemotherapy with Endoxana in radically operated bronchogenic carcinoma. European Journal of Cancer, 7, 285.

CARTER, S.K. (1972) New drugs on the horizon in bronchogenic carcinoma. Cancer, 30, 1402.

Coy, P. (1970) A randomized study of irradiation and Vinblastine in lung cancer. Cancer, 26, 803.

FreI, Emil III (1972) Combination cancer therapy. Cancer Research, 32, 2593.

Gollin, F.F., ANSField, F.J. \& Vermund, H. (1964) Clinical studies of combined chemotherapy and irradiation in inoperable bronchogenic carcinoma. American Journal of Roentgenology, 92, 88.

Green, R.A., Humphrey, E., Close, H. \& Patno, M.E. (1969) Alkylating agents in bronchogenic carcinoma. American Journal of Medicine, 46, 516.

Hansen, H.H., Muggia, F.M., Andrews, R. \& Selawry, O.S. (1972a) Intensive combined chemotherapy and radiotherapy in patients with nonresectable bronchogenic carcinoma. Cancer, 30, 315.

HANSEN, H.H.\& MugGIA, F.M. (1972b) Staging of inoperable patients with bronchogenic carcinoma with special reference to bone marrow examination and peritineoscopy. Cancer, 30, 1395.

Higgins, G.A. (1972) Use of chemotherapy as an adjuvant to surgery for bronchogenic carcinoma. Cancer, 30, 1383.

Holsti, L.R. (1972) Adjuvant chemotherapy in Modern Radiotherapy-Carcinoma of the Bronchus. Edited by Deeley, T.J., p. 222. Butterworths, London.

Medical Research Council Working Party on CarCINOMA OF THE BRONCHUS (1966) Comparative trial of surgery and radiotherapy for the primary treatment of small-celled or oat-celled carcinoma of the bronchus. Lancet, ii, 929.

Medical Research Council Working Party on CarCINOMA OF THE Bronchus (1971) Studies of Cytotoxic Chemotherapy as an adjuvant to Surgery in Carcinoma of the Bronchus. British Medical Journal, 1, 421.

Rissanen, P.M., Tikka, U. \& Holsti, L.R. (1968) Autopsy findings in lung cancer treated with megavoltage radiotherapy. Acta radiologica, therapy, 7, 443.

SelaWry, O.S. (1970) in Carbone, P.P.; Frost, J.K.; Feinstein, A.R.; Higgins, G.A. \& Selawry, O.S. Lung Cancer perspectives and prospects. Annals of Internal Medicine, 73, 1003.

\title{
Discussion
}

\author{
Chairman: DR J. R. MIKHAIL \\ (Central Middlesex Hospital and \\ Willesden Chest Clinic)
}

DR ANGEL: Could we have some of Professor Bleehen's thoughts about daily versus intermittent use of drugs. You just mentioned now cyclophosphamide $1 \cdot 3 \mathrm{~g} / \mathrm{m}^{2}$ every 3 weeks. Is this given intravenously? Would you like to develop this?

Professor BleEHEN: I would like to say something about this, because it is perhaps one useful message I could leave with you. I did touch upon this a bit when I was taking about some of the cyclophosphamide results and then showed the results where cyclophosphamide had an adverse effect. I think from what we know of cancer today it would seem likely that there is some host reaction to the tumour. Whether this is immunological or not I am not the person to argue and I do not think anyone can tell at this stage. However, if there is a host reaction it is quite possible that continuous therapy as opposed to intermittent therapy could suppress this. If you bounce the treatment in, give the body time to recover, bounce it in again, you will have far less effect on the immunity. This may be a theoretical objection to continuous therapy or not. The other advantage of intermittent therapy is that you give the normal tissues-I am not talking about immunological responses-time to recover. On the whole normal tissues will speed up their 